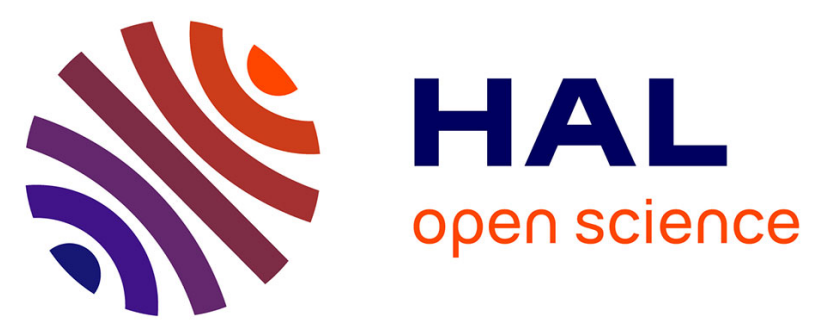

\title{
Quantitative Assessment of Motor Deficit with an Intelligent Key Object: A Pilot Study
}

Asif Hussain, Wayne Dailey, Sivakumar Balasubramanian, Nathanael Jarrasse, Samuel Kamalesh Kumar, Suresh Devasahayam, Etienne Burdet

\section{To cite this version:}

Asif Hussain, Wayne Dailey, Sivakumar Balasubramanian, Nathanael Jarrasse, Samuel Kamalesh Kumar, et al.. Quantitative Assessment of Motor Deficit with an Intelligent Key Object: A Pilot Study. 2015 IEEE International Conference on Rehabilitation Robotics (ICORR), Aug 2015, Singapore, Singapore. pp.247-252. hal-02110223

\section{HAL Id: hal-02110223 \\ https://hal.science/hal-02110223}

Submitted on 25 Apr 2019

HAL is a multi-disciplinary open access archive for the deposit and dissemination of scientific research documents, whether they are published or not. The documents may come from teaching and research institutions in France or abroad, or from public or private research centers.
L'archive ouverte pluridisciplinaire $\mathbf{H A L}$, est destinée au dépôt et à la diffusion de documents scientifiques de niveau recherche, publiés ou non, émanant des établissements d'enseignement et de recherche français ou étrangers, des laboratoires publics ou privés. 


\title{
Quantitative Assessment of Motor Deficit with an Intelligent Key Object: A Pilot Study
}

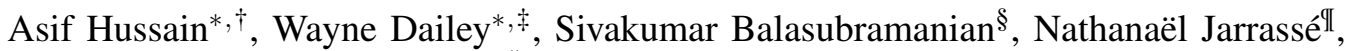 \\ Samuel Kamalesh Kumar", Suresh Devasahayam ${ }^{\S}$, and Etienne Burdet ${ }^{\ddagger}$ \\ ${ }^{*}$ Both first authors contributed equally to this work. \\ Email: ahussain@ntu.edu.sg, \{w.dailey12, e.burdet\} @imperial.ac.uk \\ ${ }^{\dagger}$ School of Mechanical and Aerospace Engineering, Nanyang Technological University, 639798 Singapore \\ ${ }^{\ddagger}$ Department of Bioengineering, Imperial College of Science, Technology and Medicine, London SW7 2AZ, UK \\ $\S^{\S}$ Department of Bioengineering, Christian Medical College, Bagayam, Vellore 632002, Tamil Nadu, India \\ IIInstitut des Systèmes Intelligents et de Robotique, Université Pierre et Marie Curie, 4 Place Jussieu, 75005 Paris, France

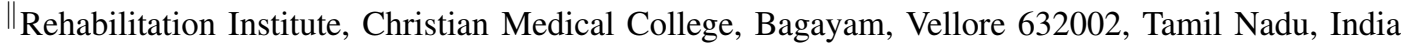

\begin{abstract}
Conventional assessment of sensorimotor functions is carried out using standard clinical scales which are subjective and insufficiently sensitive to changes in motor performance. Alternatively, sensor based systems offer a quantitative approach to motor assessment. We have designed a set of low cost, easy to use instrumented objects to assess a subject's performance during skilled tasks. In this pilot study we discuss the design of one object, the intelligent key, and describe how it can be used to assess a subject's performance during fine manipulation tasks using the proposed metrics and techniques. Three subjects with motor disability and one healthy subject participated in this study. Subjects performed insertion and rotation tasks that mimic the skills used in day to day key manipulation. A threshold detector algorithm based on Teager Energy Operator was applied to the object acceleration signal to quantify time spent struggling with the task and Spectral Arc Length was used to assess the smoothness of pronation/supination. Overall, the results indicate that increased difficulty in task performance correlates with decreased smoothness in task performance.
\end{abstract}

\section{INTRODUCTION}

Conventional assessment of motor functions is carried out by therapists using ordinal clinical scales to investigate specific aspects of a subject's motor behavior [1], [2]. Although these measures are widely accepted, standardized and validated, they are still subjective and suffer from low resolution as is typical of ordinal scales. Furthermore, the time required to perform manual assessment discourages their regular administration to track and understand motor recovery in affected population.

To address these issues, several studies have investigated the use of robotic devices for the assessment of the sensorimotor function in patients suffering from neurological diseases or injuries [3], [4], [5]. Although these devices primarily target rehabilitation, integrated sensors offer complementary functionality as a means of monitoring task performance. This approach offers a more quantitative and precise way of assessing subjects performance compared to the subjective measures currently used in clinical practice [6]. Robotic-aided assessment does not require additional time as it is performed simultaneously to regular therapy.
However, assessment is subject to the physical constraints introduced by the therapy robot, and the high costs and design complexity typically associated with these devices limits their use in clinical environment.

Sensor-based technology for the measurement and quantification of sensorimotor performance may offer an alternative solution to robotic assessment. Compact, low cost and intrinsically passive thus safe sensors (as no motor is involved) have the potential to be easily wearable, deployed in home, and installed in clinical environment [7], [8], [9]. Some of these systems are already in the market and are being used extensively in clinical environment for measuring specific sensorimotor information covering the impairment level of health and motor disability, as defined by international classification of health and disability (ICF) nomenclature [10]. For example, Jamar is one such standard tool that uses force information to determine impairment in grip strength [11]. However, limited work has been done on the design of sensor based systems to quantify a subject's ability at the activity level during tasks requiring skill. In response, we have designed a set of compact cost-effective intelligent objects using multiple sensors for assessment (and rehabilitation) of subjects performance during complex (skilled) tasks in order to quantify disability at activity level [12], [13]. The following is a brief description of designed objects:

Intelligent box (iBox), is designed to monitor pick and place and unimanual manipulation tasks. Skills assessed include the ability to lift and anticipate the amount of force required to move an object from one location to the other.

Intelligent can (iCan), is designed to monitor and facilitate rehabilitation of bimanual tasks similar to opening a can, i.e. gross power grip tasks. Skills assessed include opening a compressed fit cap.

Intelligent jar (iJar), to imitate removing a screw fit cap. Assesses the ability to manipulate objects requiring forearm pronation/supination and wrist radial/ulnar deviation, and the ability to apply enough grip force to unscrew caps.

Intelligent key (iKey), Key manipulation and operation 
of a door/car lock. Assesses the ability to perform fine finger manipulation (e.g. pinch grasp) and perform forearm pronation/supination.

This paper discusses the design of the iKey, its improvement since the previous version, and introduces a pilot study that demonstrates how sensory data can be used for assessment of fine manipulation tasks.

\section{METHODOLOGY}

\section{A. Apparatus}

The apparatus used in this study is a modified version of the Intelligent key presented in [12]. The apparatus and its modifications are explained briefly. The hardware consists of three main components: MIMATE, the keys, and a box for key insertion.

1) MIMATE: Or, Multimodal Interactive Motor Assessment and Training Environment, is a reconfigurable, wireless measurement/feedback platform designed for use as a general basis for developing training and human sensorimotor behavior assessment systems. For a full description of MIMATE, the reader is referred to [13]. In this study, a MIMATE provides a platform for feedback and data acquisition from the key and box objects explained later in the section.

2) Keys: Two types of keys were designed for the purpose of the study. Both keys resemble typical keys (dimension $60 \times 30 \mathrm{~mm}$ ). Each key consists of a cuboidal body, which the subject uses to grasp the key, and a shaft which is inserted into a keyhole. The only difference between the two keys is the design of the shaft. The first key (Key-1) has a cylindrical shaft while the second key (Key-2) has the same cylindrical shaft with an extrusion along one end. Key1 can be inserted in the keyhole in different configurations depending upon the keyhole selected. Key-2 can only be inserted in one possible way. The two keys were designed to grade the level of difficulty for key insertion. The easiest key insertion requires no specific configuration while the most difficult demands exact alignment.

A circular FSR with a sensing diameter of $12.7 \mathrm{~mm}$ is placed on one of the faces of the cuboidal part of each key as illustrated in Fig. 1 This part of the key also houses a vibrator to provide tactile sensory feedback. Both keys can be connected to the box (one at a time) through a cable to the MIMATE module. The MIMATE facilitates the acquisition of force information and can be programmed to activate or deactivate vibratory tactile feedback on the key.

3) Box: The box contains three keyholes for key insertion, an accelerometer to detect any form of interaction with the object, a potentiometer(encoder) to measure the angle, and a MIMATE module. Two keyholes are positioned on the front of the box and the third key hole is on the right side, and in theory, visually obscured from the user. Keyholes2 and 3 each consist of a circular hole for key insertion. Keyhole-1 differs slightly as it incorporates an encoder which can detect the rotation of the keyhole. Additionally, Keyhole-1 is extruded to form a plus-shaped grove which allows a limited number of configurations for key insertion,

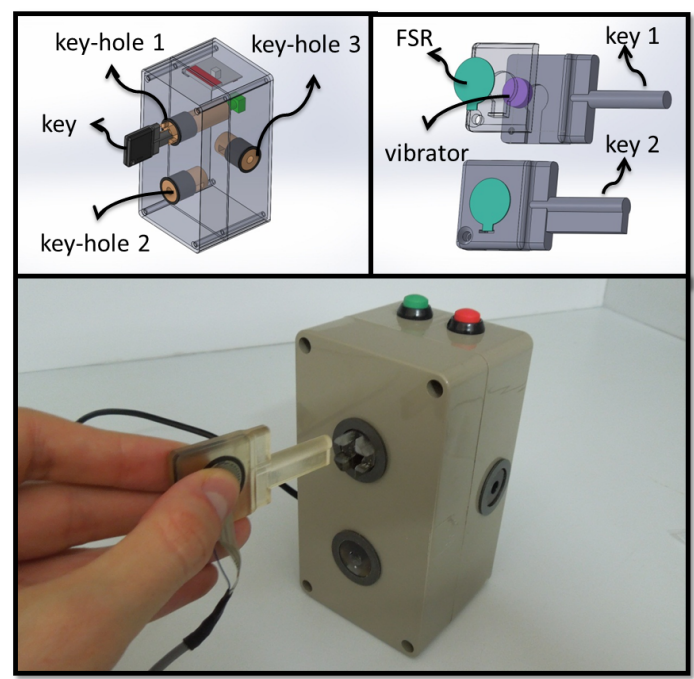

Fig. 1: Top: A 3D model of the iKey object and the design of keys. Bottom: The iKey in use.

i.e. for Key-1 only two configurations are possible, and for Key-2 only a single configuration is possible. Fig. 1 illustrates the configurations in greater detail. Each keyhole contains an internal switch to detect key insertion.

Measurements from the switches, accelerometer, encoder (potentiometer), and the key force sensor are sampled at approximately $100 \mathrm{~Hz}$ by the MIMATE. All of recorded information is transmitted wirelessly to a workstation where it is displayed for online feedback and stored for additional offline analysis.

\section{B. Experiment}

The iKey was experimentally evaluated on one healthy subject and three patients with motor deficits of the upper limb. Individuals with upper limb motor deficits who were cognitively able to understand the task and instructions were selected for the study. Patients without any functionality of the affected upper limb were excluded. Table I summarizes the characteristics of each subject. All subjects in this study were naturally right-hand dominant with right-side hemiparesis (where applicable). Recent Box and Blocks test scores were available for each of the patients involved in this study.

iKey assessment participants were seated on a chair or wheel chair (depending upon severity of impairment or injury) in a symmetrical position with the trunk extended. The iKey was placed in front of the subject, along the midline, with Keyholes- 1 and 2 facing the patient. The key appropriate for the each task (explained later in the section) was placed in front of the patient midway between the front of the iKey and the edge of table along the midline with the shank of the key pointing toward the iKey, the force sensitive side facing up, and the cable connecting the key to the box running to the right relative to the subject's perspective. Fig. 2 illustrates the positioning of the patient and the iKey object. 


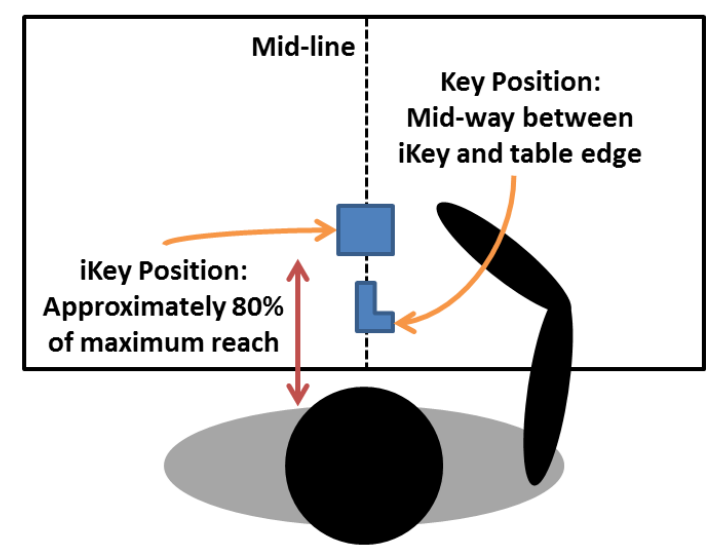

Fig. 2: Depiction of the subject and iKey placement.

The purpose and structure of the experiment was explained to the patient. A demonstration of each task was provided before starting the experiment and verbal cues were provided throughout all of the trials. Testing was conducted under the supervision of the patient's on-duty therapist. During each trial, subjects were asked to complete the following four tasks. In the case of Tasks 3 and 4, the patients were instructed to rotate $90^{\circ}$ in both directions to the best of their ability.

Task 1: Grab Key-1; Insert Key-1 into Keyhole-2 (front, bottom); Remove Key-l and place in original position.

Task 2: Grab Key-1; Insert Key-1 into Keyhole-3 (side); Remove Key-1 and place in original position.

Task 3: Grab Key-1; Insert Key-1 into Keyhole-1 (front, top); Rotate counter-clockwise (CCW); Return to center; Rotate clockwise (CW); Return to center; Remove Key-l and place in original position.

Task 4: Grab Key-2; Insert Key-2 into Keyhole-1 (front, top); Rotate counter-clockwise (CCW); Return to center; Rotate clockwise (CW); Return to center; Remove Key-2 and place in original position.

Each subject completed 3 trials, each comprised of the 4 tasks described above. The order of tasks was randomized at the beginning of each trial and verbal instructions indicating which task to complete and how to complete the task were provided. Patients were instructed to stabilize the iKey with the unaffected limb and use only the affected limb for key manipulation. Keys- 1 and 2 were interchanged by the experimenter at the appropriate time in accordance with the task order for each trial.

\section{Data Analysis}

The initial two seconds of data from each trial were ignored and the standard deviation of the acceleration magnitude was calculated between $t=[2,4] \mathrm{s}$ relative to the start of the trial. The following measures use the magnitude-mean acceleration data, calculated from the $x, y$ and $z$ acceleration components, for the struggle time metric developed later in this section.

Encoder measurement data of Keyhole-1 angular position was segmented into four sections during rotation tasks (Tasks 3 and 4). Each segment was processed independently. Angular position data for key rotation in Tasks 3 and 4 were grouped together because rotation is independent of key type once insertion is achieved.

1) Struggle Time: Struggle time was developed as a metric for quantifying the difficulty in completing any of the four tasks considered in this study. It is assumed that periods of heightened acceleration activity (e.g. when the box is disturbed during poorly coordinated key insertion) correlates with greater difficulty performing the task for the subject. The acceleration data was segmented by task and the struggle time for each was calculated by converting the mean magnitude acceleration to Teager Energy and applying a threshold detector. This measure should be similar to the total time required to complete the exercise, but also identifies challenging components within the task. For example, individuals with bilateral coordination deficits may struggle during the bimanual grasping phase, but otherwise perform key insertion with ease. Further, total task time, which is essentially a measure of speed, does not necessarily correspond to quality of performance in fine motor tasks.

Teager Energy: The Teager Energy Operator (TEO) is a common signal processing technique that provides an estimate of the instantaneous energy of an arbitrary signal [14], [15]. The definition of the discrete TEO [14] is given by:

$$
\Psi\left[s_{n}\right]=s_{n}^{2}-s_{n-1} s_{n+1}
$$

where $s_{n}$ is an arbitrary discrete time signal.

Teager Threshold Detector: A threshold detector was designed for analysis of the Teager Energy (TE) of acceleration to identify periods of pronounced kinetic activity. The threshold detector algorithm operates on both TE magnitude and duration. Including duration ensures that isolated spikes are excluded from the metric. The TE standard deviation between $t=[2,4] \mathrm{s}$, i.e. the initial rest period, was calculated for each trial and used as the baseline for the threshold. The magnitude criterion was satisfied when the magnitude of the TE exceeded 7 times the baseline standard deviation. The threshold was maintained in the active state until the threshold criterion was no longer satisfied at any point within a period of $0.5 \mathrm{~s}$. Finally, threshold pulses lasting $0.2 \mathrm{~s}$ or less were removed.

2) Smoothness of Key Rotation: The smoothness of the pronation/supination key rotation movements of Tasks 3 and 4 were assessed by breaking both tasks into four subtasks: $\mathrm{CCW}$ rotation (pronation) from center, $\mathrm{CW}$ rotation (supination) toward center, $\mathrm{CW}$ rotation from center, and $\mathrm{CCW}$ rotation toward center. A cubic spline fit was applied to the subtasks angular position and the data was up-sampled to $1000 \mathrm{~Hz}$. Angular velocity was then calculated by numerical differentiation. 
TABLE I: Summary of the subjects and relevant clinical information.

\begin{tabular}{|c|c|c|c|c|c|c|}
\hline Subject & Sex & Pathology & Other Conditions & Box and Blocks Scores & Dominant Hand & Affected Hand \\
\hline 1 & Male & Healthy & None & N/A & $\mathrm{R}$ & N/A \\
\hline 2 & Female & $\begin{array}{lll}\begin{array}{l}\text { Peritoneovenous shunt, } \\
\text { weakness (full) }\end{array} & \\
\text { (R) }\end{array}$ & None & R:26 L:52 & $\mathrm{R}$ & R-(full) \\
\hline 3 & Male & $\begin{array}{l}\text { Traumatic brain injury, (R) } \\
\text { weakness (full), (L) weakness } \\
\text { (partial) }\end{array}$ & $\begin{array}{l}\text { (R) radial ulnar fraction, } \\
\text { bi-lateral coordination is- } \\
\text { sues }\end{array}$ & R:48 L:42 & $\mathrm{R}$ & $\begin{array}{l}\text { R-(full), } \\
\text { (partial) }\end{array}$ \\
\hline 4 & Male & Acute encephalomyelitis & None & R:18 L:32 & $\mathrm{R}$ & R-(full) \\
\hline
\end{tabular}

Spectral Arc Length: A recent study suggests that existing smoothness measures such as jerk are not dimensionless and respond inconsistently to changes in smoothness [16]. The Spectral Arc Length (SAL) smoothness metric developed in [16] is a robust, dimensionless measure of the length, and thus the complexity, of a frequency spectrum curve over the bandwidth of interest. SAL has been validated as a consistent method for quantifying the movement smoothness. The SAL metrics was applied to the angular velocity of the subtasks in each trial and is defined by:

$$
\begin{array}{r}
\eta_{s a l} \stackrel{\text { def }}{=}-\int_{0}^{\omega_{c}} \sqrt{\left(\frac{1}{\omega_{c}}\right)^{2}+\left(\frac{\mathrm{d} \hat{S}(\omega)}{\mathrm{d} \omega}\right)^{2}} \mathrm{~d} \omega, \\
\hat{S}(\omega) \stackrel{\text { def }}{=} \frac{S(\omega)}{S(0)}
\end{array}
$$

where $S(\omega)$ is the Fourier magnitude spectrum of velocity speed signal $s(t)$ and $\left[0, \omega_{c}\right]$ is the frequency band occupied by the movement of interest. For this study, the upper frequency bound was set to $20 \mathrm{~Hz}$ as this is typically sufficient to capture normal and abnormal human motions [16]. Smoother movements will have SAL values closer to zero, and increasingly negative values as the number of sub movements increases.

\section{RESULTS}

All participants were able to complete the tasks with the exception of Subject 4, who was unable to complete the tasks without assistance from a therapist and struggled to follow the instructions properly. The data of Subject 4 was excluded from the results in this study.

Fig. 3 demonstrates typical data for Task 4 for the healthy and one affected subject. The top plots illustrate the angular position of Keyhole-1 and the force detected by Key- 2 while the bottom plots provide the TE of the mean magnitude acceleration and the resulting threshold detector.

\section{A. Struggle Time}

Fig. 4 shows struggle time for each task. The results indicate that overall struggle time for the healthy subject is lower than that of the affected population. Among the different tasks, struggle time is higher for tasks in which the subject had to orient the key appropriately for insertion into the keyhole, i.e. struggle time is higher for Tasks 3 and 4 compared to Tasks 1 and 2. This was expected as these tasks involve both key insertion and rotation and thus are more difficult to perform. It is important to note that examining individual hand scores within patients may not provide an accurate representation of impairment for bimanual tasks such as those encountered frequently in daily life.

\section{B. Smoothness of Key Rotation}

The results of the smoothness measure also indicate an increase in smoothness of key rotation as the level of disability decreases, i.e. the subject with higher box and block score has higher value of smoothness that the subject with the lower score. Subject 3 was rated with a relatively high box and blocks score so it is reasonable that the corresponding smoothness was similar to that of Subject 1 . Fig. 5 shows the combined results of rotation during Tasks 3 and 4 . Since Tasks 3 and 4 differ only in key insertion, the key rotation subtasks of both cases are grouped in the assessment of smoothness. Subject 2 demonstrated relatively low SAL values with higher variability for the "Center to Left" and "Centre to Right" key rotations. This could be due to greater difficulty in pronation than supination for this particular individual.

\section{DISCUSSION AND FUTURE WORK}

In this study we describe the design of the modified version of the iKey and demonstrate its use in the assessment of fine manipulation tasks by examining subject performance in activities similar to key handling and manipulation. Three parameters were considered for the purpose of assessment: 1) successful key insertion, the ability of subject to successfully insert the key inside the three different keyholes which varied by type/configuration, 2) struggle time, the amount of time subjects struggled to perform a task as a result of wrong orientation, positioning, etc. and 3) smoothness, the ability of the subject to efficiently perform the defined pronation and supination tasks. Although the number of subjects tested is limited, the results indicate that these measures have potential use for quantitatively grading a subject's performance. In this case all subjects were able to place the key in the key holes. In general, subjects with severe disability may have more difficulty placing the key in the keyhole, especially for cases in which the key requires a specific orientation.

Struggle time for the healthy subject is always less than that of the unhealthy subjects and increases for tasks involving specific orientation of the key; subjects with disability experience difficulty in orienting and positioning the key in front of the hole and the chance of striking the wall of the box will be greater, as is evident in the data. This measure may be a better indicator of motor performance than total 

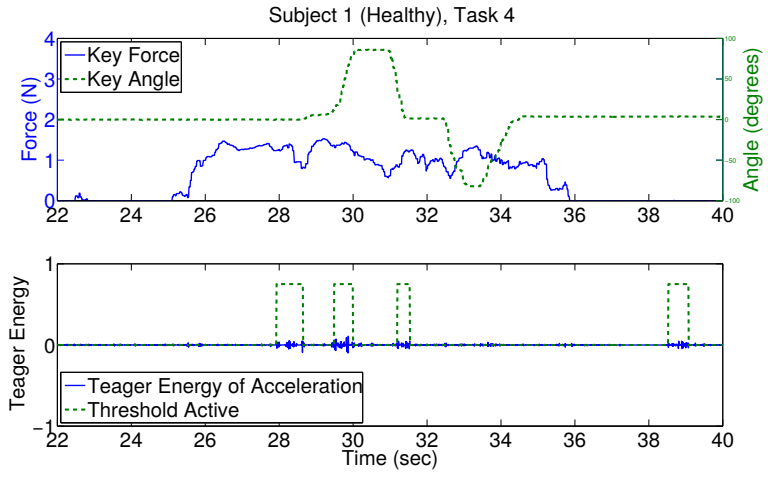

(a) Healthy
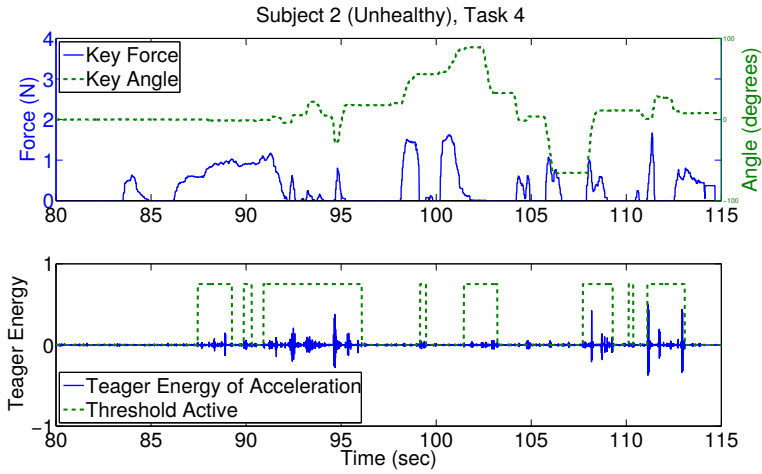

(b) Affected

Fig. 3: Struggle time for healthy subject is less compared to affected population; (a) shows task performance of healthy subject and calculated struggle time and (b) shows task performance of one impaired subject and estimated struggle time.

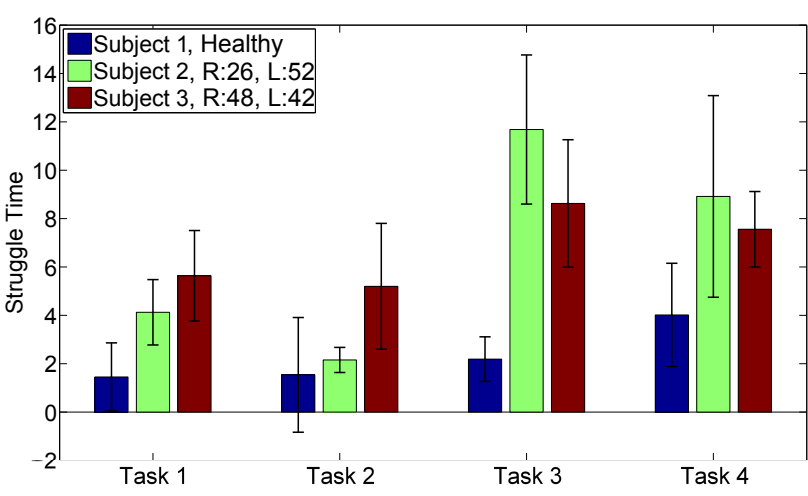

Fig. 4: Bar plot of struggle time of analyzed population.

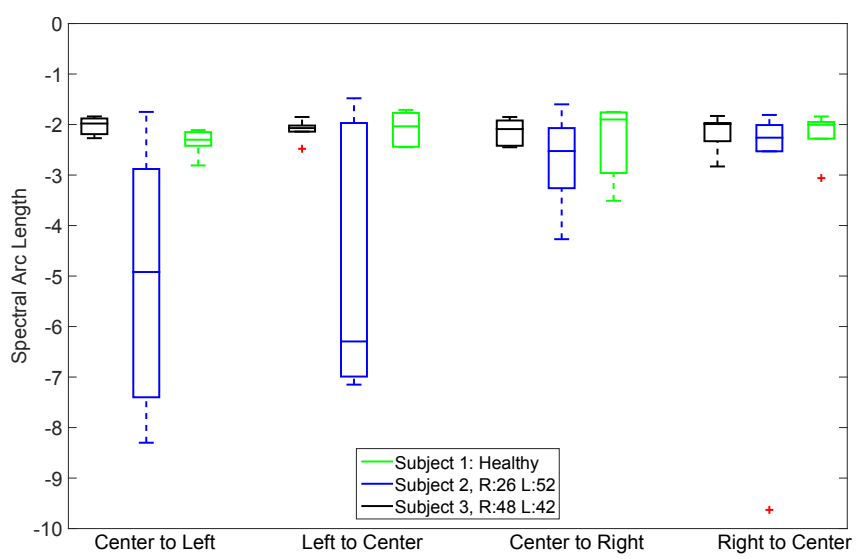

Fig. 5: Smoother motions are indicated by spectral arc length values closer to zero. Movement is less smooth for subjects with higher degree of disability (see box and blocks test score). time required to complete the task. Considering only the total completion time imposes a time constraint on the task rather emphasizing quality and control in task execution. However, additional evidence is required to fully justify this hypothesis.

Smoothness has been used as a measure of task performance in number of studies [5], [17], [18], [19]. In general, increased smoothness is observed for healthy subjects. We have used the Spectral Arc Length proposed by Balasubramanian et al. [16] as it has been shown to be more robust than other measures proposed. Our findings are in agreement with the previous results, i.e. smoothness increases with decrease in disability.

The current study was open to subjects with any form of upper limb motor deficits as the aim was to simply investigate the principle of working. We aim to do a broader study with larger set of patients, but with a more refined inclusion criteria to investigate reliability and sensitivity of the measures proposed. The current study did not assess force information from the iKey as patients often grab the key without touching the force sensitive surface. This issue needs to be addressed in future studies.

Multivariate analysis of these measures can be used to help grade a subject's performance, but this remains to be investigated. The results indicate that acceleration data recorded from the box can be used to estimate the subject's difficulty using the struggle time metric and Spectral Arc Length can be applied to key rotation tasks to assess smoothness in pronation/supination. This study provides examples of quantitative metrics that can be developed using sensorized objects. Many other potential metrics such as detailed analysis of interaction force as demonstrated in [20] should be explored in future work. The resolution and reliability of these measures must be evaluated in larger studies with more participants. It may also be possible to establish healthy baseline values using a large control population. However, devices such as the iKey are promising devices for quantitative assessment and the development of novel motor assessment metrics. 


\section{ACKNOWLEDGMENT}

This publication was made possible by UK-UKIERI IND/CONT/E/1172/182, FP7-PEOPLE-2012-ITN project No 317488: CONTEST, and the Whitaker International Program. The MIMATE module was designed by Dr. Nicholas Roach. The authors would like to thank Christian Medical College (Vellore, India), Mr. Akhil Mohan, and the clinicians for helping with patient recruitment and experimental supervision.

\section{REFERENCES}

[1] D. Gladstone, C. Danells, and S. Black, "The Fugl-Meyer assessment of motor recovery after stroke: a critical review of its measurement properties," Neurorehabilitation and Neural Repair, vol. 16, no. 3, pp. 232-240, Sep. 2002.

[2] C. Wu, T. Fu, K. Lin, C. Feng, and K. Hsieh, "Assessing the streamlined Wolf Motor Function Test as an outcome measure for stroke rehabilitation," Neurorehabilitation and neural repair, vol. 25 no. 2, pp. 194-199, Feb. 2011.

[3] M. D. Ellis, T. Sukal-Moulton, and J. P. A. Dewald, "Progressive Shoulder Abduction Loading is a Crucial Element of Arm Rehabilitation in Chronic Stroke,' Neurorehabilitation and Neural Repair, vol. 23, no. 8, pp. 862-869, Oct. 2009.

[4] O. Lambercy, L. Dovat, H. Yun, S. K. Wee, C. Kuah, K. Chua, R. Gassert, T. Milner, T. C. Leong, and E. Burdet, "Robotic assessment of hand function with the hapticknob," in Proceedings of the 4th International Convention on Rehabilitation Engineering \& Assistive Technology, ser. iCREATe '10. Kaki Bukit TechPark II,, Singapore: Singapore Therapeutic, Assistive \& Rehabilitative Technologies (START) Centre, 2010, pp. 33:1-33:4.

[5] C. Bosecker and L. Dipietro, "Kinematic robot-based evaluation scales and clinical counterparts to measure upper limb motor performance in patients with chronic stroke," Neurorehabilitation and neural repair, vol. 24, no. 1, pp. 62-69, Jan. 2010.

[6] O. Celik, M. K. O’Malley, C. Boake, H. S. Levin, N. Yozbatiran, and T. A. Reistetter, "Normalized Movement Quality Measures for Therapeutic Robots Strongly Correlate With Clinical Motor Impairment Measures," IEEE Transactions on Neural Systems and Rehabilitation Engineering, vol. 18, no. 4, pp. 433-444, Aug. 2010. [Online]. Available: http://ieeexplore.ieee.org/lpdocs/epic03/wrapper.htm?arnumber=5446376

[7] J. Kowalczewski, S. L. Chong, M. Galea, and A. Prochazka, "InHome Tele-Rehabilitation Improves Tetraplegic Hand Function,' Neurorehabilitation and Neural Repair, Mar. 2011.

[8] P. Bonato, "Advances in wearable technology and its medical applications," Engineering in Medicine and Biology Society, pp. 2021-2024, Sep. 2010.

[9] P. Bonato, "Advances in wearable technology and applications in physical medicine and rehabilitation," Journal of NeuroEngineering and Rehabilitation, vol. 2, no. 1, p. 2, 2005.

[10] A. M. Jette, "Toward a Common Language for Function, Disability, and Health," Physical Therapy, vol. 86, no. 5, pp. 726-734, May 2006.

[11] L. Ashton and S. Myers, "Serial grip strength testing-its role in assessment of wrist and hand disability," The Internet Journal of Surgery, vol. 5, no. 2, 2004.

[12] A. Hussain, N. Roach, S. Balasubramanian, and E. Burdet, "A modular sensor-based system for the rehabilitation and assessment of manipulation," in Haptics Symposium (HAPTICS), 2012 IEEE. IEEE, 2012, pp. 247-254.

[13] N. Roach, A. Hussain, and E. Burdet, "Learning to Design Rehabilitation Devices Through the H-CARD Course: Project-Based Learning of Rehabilitation Technology Design," IEEE Pulse, vol. 3, no. 6 , pp. $51-58$, Nov. 2012.

[14] F. Jabloun, A. Cetin, and E. Erzin, "Teager energy based feature parameters for speech recognition in car noise," Signal Processing Letters, IEEE, vol. 6, no. 10, pp. 259 -261, oct. 1999.
[15] E. Kvedalen, "Signal processing using the Teager energy operator and other nonlinear operators," Master, University of Oslo Department of Informatics, pp. 33:1-33:4, 2003.

[16] S. Balasubramanian, A. Melendez-Calderon, and E. Burdet, "A Robust and Sensitive Metric for Quantifying Movement Smoothness," IEEE Transactions on Biomedical Engineering, vol. 59, no. 8, pp. 2126 -2136, Aug. 2012.

[17] B. Rohrer, S. Fasoli, H. I. Krebs, R. Hughes, B. Volpe, W. R. Frontera, J. Stein, and N. Hogan, "Movement Smoothness Changes during Stroke Recovery," The Journal of Neuroscience, vol. 22, no. 18 , pp. $8297-8304$, Sep. 2002

[18] R. Colombo and F. Pisano, "Robotic techniques for upper limb evaluation and rehabilitation of stroke patients," Neural Systems and Rehabilitation Engineering, IEEE Transactions on, vol. 13, no. 3, pp. $311-324$, Sep. 2005.

[19] S. Balasubramanian and R. Colombo, "Robotic Assessment of Upper Limb Motor Function After Stroke," American Journal of Physical Medicine \& Rehabilitation, vol. 91, pp. S255-S269, Nov. 2012.

[20] N. Jarrasse, M. Kuhne, N. Roach, A. Hussain, S. Balasubramanian, E. Burdet, and A. Roby-Brami, "Analysis of grasping strategies and function in hemiparetic patients using an instrumented object," in $R e$ habilitation Robotics (ICORR), 2013 IEEE International Conference on. IEEE, 2013, pp. 1-8. 